processed through Spectronaut 14 software for spectral library building, protein identification and quantification. Differentially expressed proteins were identified based on an observed fold change of $\geq 1.5$ or $\leq-1.5$ and q-value $\leq 0.005$. Pathway analysis was performed using Ingenuity Pathway Analysis (IPA) software.

Results and Conclusions/Implications Label-free MS analysis led to over 4,000 protein identifications, with 3,484 proteins commonly identified across all patient samples. Over 1,000 significantly differentially expressed protein candidates were identified for comparisons between NF and DCM, HOCM or ISCM. DCM-specific protein changes were strongly associated with glutamine biosynthesis, HOCM-specific protein changes were strongly associated with LXR/RXR Activation, while ISCM-specific protein changes were most associated with tryptophan degradation pathways. DCM vs NF, ISCM vs NF and HOCM v NF had shared differentially expressed proteins that were also significantly altered at gene level $(n=106)$. Canonical pathway analysis revealed that Choline Degradation and Lysine Degradation pathways were most strongly associated with these candidates. Expression changes for some of the top over- and under-expressed HF candidates were validated in an independent replicate dataset (PXD008934) [2]. This represents one of the largest and deepest proteomic datasets for myocardial tissue reported to date. The dataset, which compliments existing transcriptomic data for these samples, has highlighted a number of significant proteins associated with different underlying aetiologies of HF. Prognosis for HF differs depending on the aetiology from which it arises. Hence, the dataset here will help in further understanding the pathogenesis of the disease, leading towards more personalised treatment.

Conflict of Interest N/A

\section{CLINICAL, PHYSICAL, COGNITIVE AND SOCIAL FRAILTY IN PATIENTS WITH CHRONIC HEART FAILURE: PREVALENCE AND ASSOCIATIONS WITH OUTCOME}

${ }^{1}$ Shirley Sze, ${ }^{2}$ Pierpaolo Pellicori, ${ }^{3}$ Jufen Zhang, ${ }^{4}$ Joan Weston, ${ }^{5}$ lain Squire, ${ }^{6}$ Andrew Clark. ${ }^{1}$ Leicester University Hospitals NHS Trust, Leicester, UK; ${ }^{2}$ University of glasgow; ${ }^{3}$ Anglia Ruskin University; ${ }^{4}$ University of Hull; ${ }^{5}$ University of Leicester; ${ }^{6}$ Hull York Medical School

\subsection{6/heartjnl-2021-BCS.113}

Background Recently, the American College of Cardiology (ACC) and the Heart Failure Association (HFA) of the European Society of Cardiology (ESC) proposed a four-domain approach to assess frailty in patients with heart failure (HF), to tailor treatment and potentially improve outcomes.Aim: To study the prevalence and prognostic value of four different types of frailty deficits: clinical, physical, cognitive and social frailty in ambulatory patients with HF.

Methods We assessed prospectively consecutive patients attending a routine follow-up visit. Patients with $\geq 5$ non-HF comorbidities were classified as having a clinical deficit. Those who scored $\geq 3$ using the Fried criteria were classified as having a physical deficit. Those who failed to complete a clock test accurately were classified as having a cognitive deficit. Those who lived alone or in a residential home were classified as having a social deficit.

Results 467 patients (67\% male, median (25th-75th centile) age 76 (69-82) years, median (25th-75th centile) NT-proBNP 1156 (469-2463) ng/L) were enrolled. 65\% of patients had clinical deficits, 52\% had a physical deficit, 39\% had a social

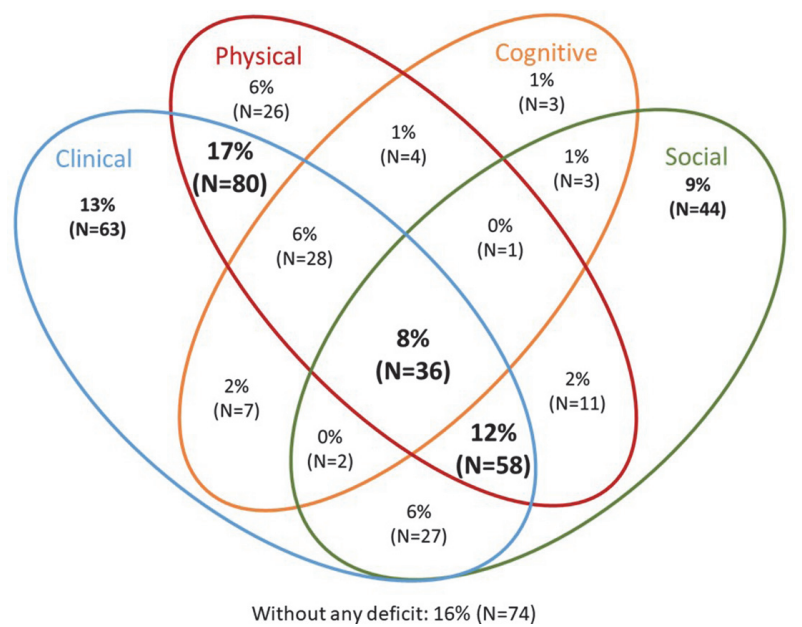

Abstract 114 Figure 1
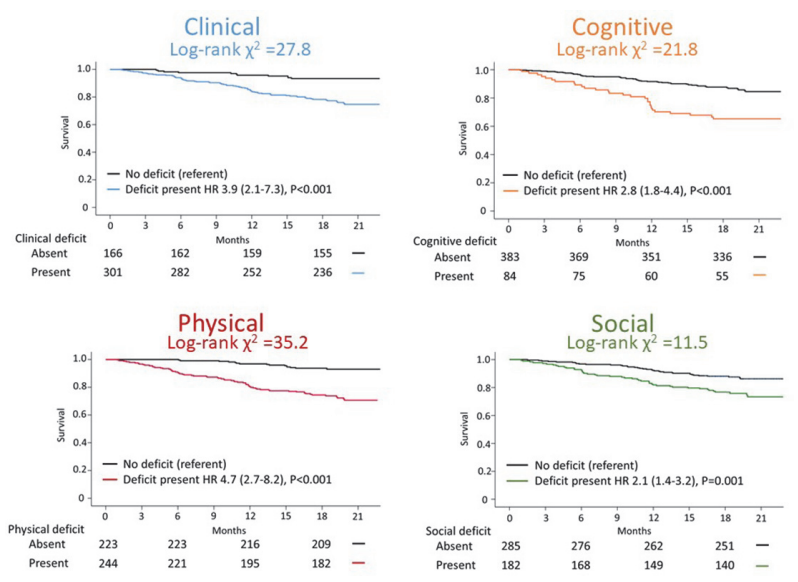

Abstract 114 Figure 2

deficit and $18 \%$ had a cognitive deficit. $28 \%$ had 2, $19 \%$ had $3,8 \%$ of patients had all 4 deficits; $16 \%$ had none. (Figure 1). During a median follow-up of 554 days, 82 patients died. The presence of any frailty deficit was associated with increased risk of mortality. (Figure 2) Patients with all 4 frailty deficits have a 15 -fold increased risk of mortality compared to patients with no frailty deficit. A base model (including age, body mass index, NYHA class and log [NT-proBNP]) for predicting mortality at 1 year achieved a C-statistic of 0.78 . Addition of all four deficits improved the performance of the base model (C-statistic $=0.82)$.

Conclusion Clinical, physical, cognitive and social deficits are common in patients with $\mathrm{HF}$ and are associated with a poor outcome. Future studies should evaluate how to optimise care for frail patients with HF using a domain management model. Conflict of Interest none

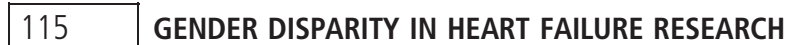

Holly Morgan, Aish Sinha, Divaka Perera. King's College London, London, UK

\subsection{6/heartjnl-2021-BCS.114}

Background Cardiovascular disease is one of the leading causes of mortality in women, accounting for $49 \%$ of all deaths, 